\title{
Ultrasound tracked motion compensated focused ultrasound system evaluated on ex vivo ovine livers
}

\author{
Jan Strehlow ${ }^{1 *}$, Xu Xiao ${ }^{2}$, Michael Schwenke ${ }^{1}$, loannis Karakitsios ${ }^{3}$, Markus Domschke $^{3}$, Senay Mihcin ${ }^{3}$, Yoav Levy ${ }^{4}$, \\ Tobias Preusser ${ }^{5}$, Andreas Melzer ${ }^{3}$
}

From Current and Future Applications of Focused Ultrasound 2014. 4th International Symposium Washington, D.C, USA. 12-16 October 2014

\section{Background/introduction}

The application of FUS in abdominal organs such as the liver or the kidneys is impeded by a number of complications. One of the most challenging is organ motion due to breathing. To achieve ablation in a target within a moving organ the FUS system has to be steered to focus on the same anatomical position. We present a prototypical system that tracks the motion of an ex vivo ovine liver via diagnostic ultrasound (US) and adjusts the focal spot to a fixed anatomical position.

\section{Methods}

Our setup consists of four systems: An organ in breathing-like motion is modeled by a robotic arm periodically moving a fresh ovine liver in water tank. The liver motion is assumed to be unknown, its movement range is $20 \mathrm{~mm}$, and one cycle takes approximately 8 seconds. A diagnostic US system is used to track vessels in the liver (Figure 1). Real time tracking positions are send to a therapy control system that calculates the position of a target using a linear motion model. The therapy control adjusts the focal spot of a steerable FUS system to match the computed target position. Since an active FUS will render our US-tracking images unusable the FUS is pulsed, leaving short imaging windows for tracking. Temperature is assessed by a thermocouple inserted into the liver. The target area is defined in vicinity of the thermocouple. Temperature curves of a moving and a static organ are acquired for different update rates and different output powers of the FUS system. The temperature curves of static and moving scenarios are compared to assess the ability of the system to compensate motion.

\section{Results and conclusions}

The US-tracking of the organ works reliably when FUS was switched off for at least $80 \mathrm{~ms}$ with $2-4 \mathrm{~Hz}$. The output power of the FUS system does not influence the reliability of the system. Temperature curves for the static and moving scenarios show only minor differences. Figure 2 shows the temperature curves acquired by sonication with a) $15 \mathrm{~W}$, b) $30 \mathrm{~W}$, and c) $45 \mathrm{~W}$ for $20 \mathrm{sec}-$ onds with a sonication update rate of $9 \mathrm{~Hz}$ and $3 \mathrm{~Hz}$ US-tracking. The average temperature differences are a) $\left.0.33{ }^{\circ} \mathrm{C} \mathrm{b}\right) 0.56{ }^{\circ} \mathrm{C}$, and c) $1.0{ }^{\circ} \mathrm{C}$. The area under the curve differs by a) $14.09 \%$, b) $1.17 \%$, and c) $0.99 \%$ (static scenario as reference). Our US-tracked steered FUS system can compensates unknown motion that is similar to the one induced by respiration. In our phantom a linear motion model was sufficient to compute the target position. For in vivo experiments this motion model has to be changed to a deformable one. The system, however, demonstrates the feasibility of US tracked steered FUS.

\section{Acknowledgements (Funding) \\ The research leading to these results has received funding from the European Community's Seventh Framework Programme FP7/2007-2013 under grant agreement $n^{\circ} 611889$. Parts of the research were supported by the the University of Dundee, a registered Scottish Charity, No: SC015096}

\section{Authors' details}

${ }^{1}$ Fraunhofer MEVIS, Bremen, Germany. ${ }^{2}$ Institute for Medical Science and Technology, Dundee, United Kingdom. ${ }^{3}$ University of Dundee, Dundee, United Kingdom. ${ }^{4}$ InSightec Ltd, Tirat Carmel, Israel. ${ }^{5}$ Fraunhofer MEVIS/ Jacobs University Bremen, Bremen, Germany.

${ }^{1}$ Fraunhofer MEVIS, Bremen, Germany

Full list of author information is available at the end of the article 


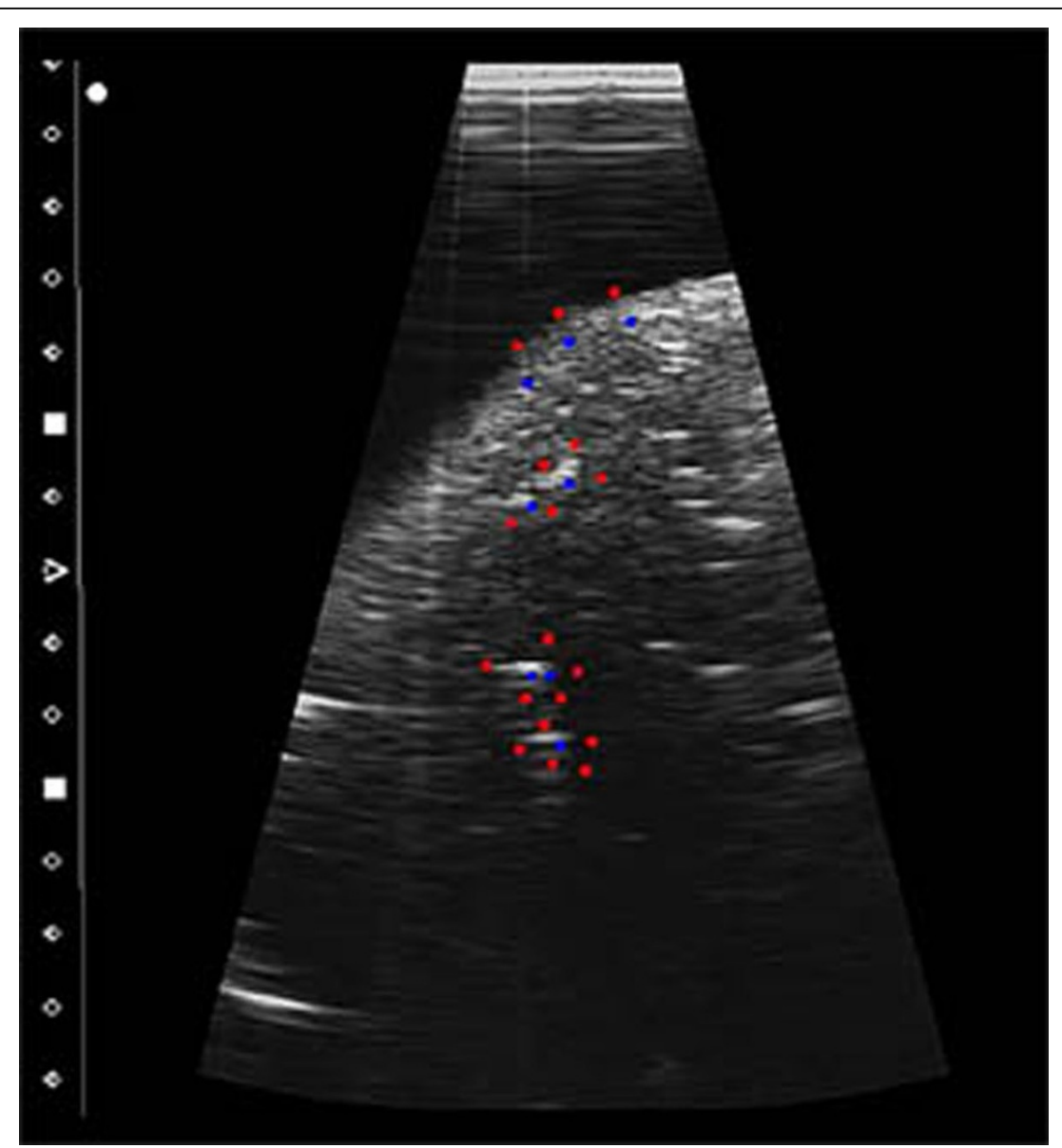

Figure 1 Liver vessels used as tracking features to assess organ motion

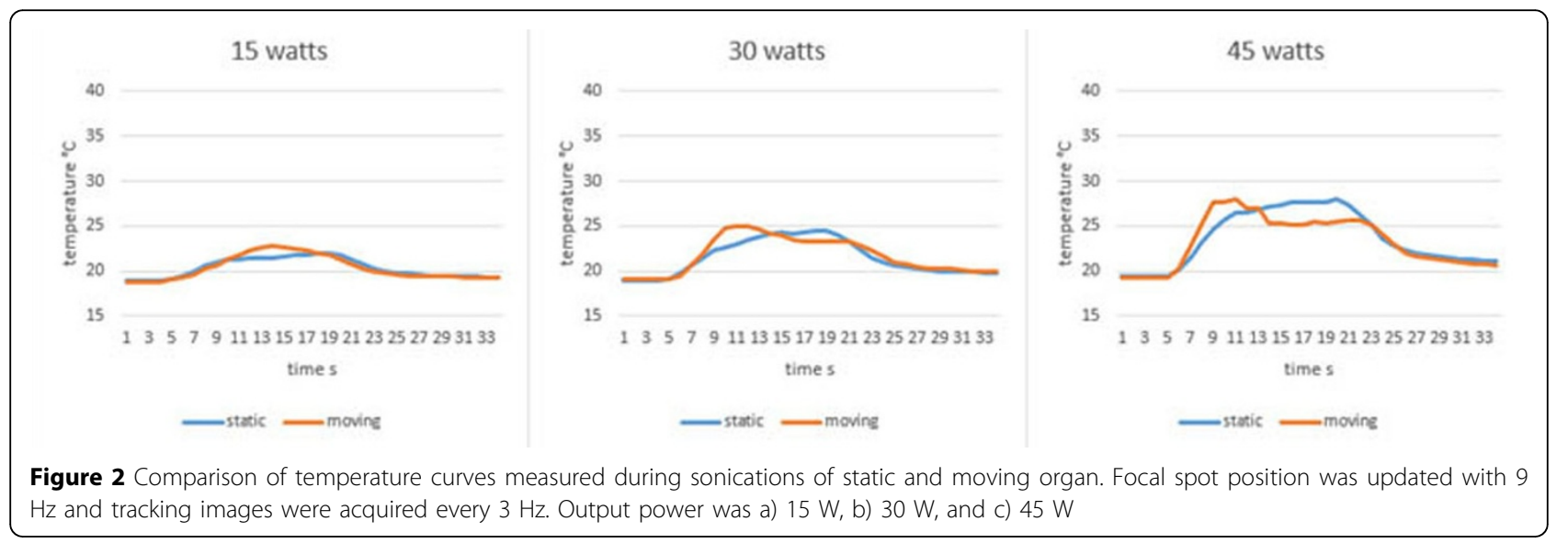

Published: 30 June 2015

doi:10.1186/2050-5736-3-S1-P52

Cite this article as: Strehlow et al:: Ultrasound tracked motion

compensated focused ultrasound system evaluated on ex vivo ovine

livers. Journal of Therapeutic Ultrasound 2015 3(Suppl 1):P52. 